\title{
Quasinormal Modes and Hawking radiation of a Reissner-Nordström black hole surrounded by quintessence
}

\author{
Mahamat Saleh ${ }^{1} \cdot$ Bouetou Bouetou Thomas ${ }^{2} \cdot$ \\ Timoleon Crepin Kofane
}

\begin{abstract}
We investigate quasinormal modes(QNMs) and Hawking radiation of a Reissner-Nordström black hole surrounded by quintessence. The Wentzel-KramersBrillouin (WKB) method is used to evaluate the QNMs and the rate of radiation. The results show that due to the interaction of the quintessence with the background metric, the QNMs of the black hole damp more slowly when increasing the density of quintessence and the black hole radiates at slower rate.
\end{abstract}

Keywords Quasinormal Modes; Hawking radiation; WKB approximation

\section{Introduction}

The investigation of the general properties of black holes has attracted considerable interest. It is well known that when perturbing a black hole, it undergoes oscillations which are characterized by some complex eigenvalues, with negative imaginary parts, of the wave equations called quasinormal frequencies. Their real parts represent the oscillation frequencies, while the imaginary ones determine the damping rates of modes. QNMs of black holes have been an intriguing subject of discussion for the last few decades(Iver \& Will (1987); Kokkotas \& Schutz (1988); Seidel \& Iyer (1990); Kokkotas (1993); Cardoso et al

Mahamat Saleh

Bouetou Bouetou Thomas

Timoleon Crepin Kofane

Department of Physics, Faculty of Science, University of Yaounde I, P.O. Box 812, Cameroon

${ }^{1}$ Department of Physics, Higher Teachers' Training College, University of Maroua, P.O. Box 55 Maroua, Cameroon

${ }^{2}$ Ecole Nationale Supérieure Polytechnique, University of Yaounde I, P.O. Box 8390, Cameroon
(2003); Zhang \& Gui (2006); Zhang et al. (2007); Mahamat et al. (2009)). On the other hand, Hawking radiation from black holes is one of the most striking effects that is known, or at least widely agreed to arise from combination of quantum mechanics and general relativity. As one of the most important achievements of quantum field theory in curved spacetime, the discovery of hawking radiation supported these ideas availably which showed that a classical black hole could radiate thermal spectrum of particles. Hawking and Ellis (Hawking \& Ellis (1973)) evoke the possible origin of radiation to be a black body radiation left over from a hot early stage of the universe, the result of superposition of a very large number of very distant unsolved discrete sources or intergalactic grains which thermalize other forms of radiations. The Hawking radiation for the Reissner-Nordström black hole is widely studied in literature(Jiang \& Wu (2006); Goncharov \& Firsova (2010); Zhai \& Liu (2010); Zhao et al. (2010)).

There has been growing observational evidence showing that our universe is accelerated expanding driven by a yet unknown dark energy. Recent results from the cosmic microwave background(CMB) combined with supernova of type Ia, large-scale structure (cosmic shear) and galaxy cluster abundances show that our universe is dominated by a mysterious dark lergy with negative pressure $(\sim 70 \%)$ and contains cold dark matter with negligible pressure $(\sim 25 \%)$, the ordinary baryonic matter makes up 5\%(Mukanov (2005)). Dark energy can be studied by its influence on the expansion of the universe as well as on the growth of the large scale structure. Cosmological models with a dark energy fluid with an equation of state parameter $\omega_{q}$ close to -1 are favored by combining recent $\mathrm{CMB}$, supernova and baryon acoustic oscillations data, suggesting that the Hubble expansion accelerates in the current cosmic epoch. There are several types of models of dark energy such 
as the cosmological constant Cardenas et al. (2003)), quintessence Varun \& Wang (2000); Kiselev (2003); Shuang-Yang (2008)), phantom(Kunz \& Domenico (2006) k-essence(Yang \& Gao (2009)), and quintom(Guo et al. (2005); Xia et al. (2006)) models. For quintessence, the equation of state parameter is in the range of $-1 \leq \omega_{q} \leq-1 / 3$. Recently, Kiselev (2003) considered Einstein's field equation surrounded by quintessential matter and obtained a new solution dependent on state parameter $\omega_{q}$ of the quintessence. Hod \& Piran (1998) investigated the late-time evolution of charged gravitational collapse and decay of charged scalar hair. Konoplya (2002) investigated the decay of charged scalar field in the Reissner-Nordström black hole background. In this paper, a Reissner-Nordström black hole surrounded by quintessence is considered to investigate QNMs and Hawking radiation including the influence of the quintessence on them.

The paper is organized as follows. In section 2 , we derive the wave equation of a scalar perturbation in the Reissner-Nordström background surrounded by quintessence. In section 3 , we evaluate the QN frequencies of the scalar perturbation by using the third order WKB approximation method. In section 4. Hawking radiation is investigated. The last section is devoted to a summary and conclusion.

\section{Scalar field perturbation}

For the Reissner-Nordström black hole, the metric is given by:

$d s^{2}=-\left(1-\frac{2 M}{r}+\frac{Q^{2}}{r^{2}}\right) d t^{2}+\left(1-\frac{2 M}{r}+\frac{Q^{2}}{r^{2}}\right)^{-1} d r^{2}+r^{2} d \Omega^{2}$

where $M$ is the black hole mass and $Q$, the charge of the black hole.

Due to the interaction of the quintessence with the spacetime, the background metric transforms to (Mahamat et al. (2009))

$d s^{2}=-f(r) d t^{2}+f(r)^{-1} d r^{2}-r^{2} d \Omega^{2}$,

with $f(r)=\left(1-\frac{2 M}{r}+\frac{Q^{2}}{r^{2}}-\frac{c}{r^{3 \omega_{q}+1}}\right), \omega_{q}$ is the quintessential state parameter, $c$ the normalization factor related to the density of quintessence, $\rho_{q}=-\frac{c}{2} \frac{3 \omega_{q}}{r^{3\left(\omega_{q}+1\right)}}$.

Using the tortoise coordinate $r_{*}$ defined by $d r_{*}=$ $\left(1-\frac{2 M}{r}+\frac{Q^{2}}{r^{2}}-\frac{c}{r^{3 \omega_{q}+1}}\right)^{-1} d r$, the metric can be rewritten as:

$d s^{2}=f(r)\left(-d t^{2}+d r_{*}^{2}\right)-r^{2}\left(d \theta^{2}+\sin ^{2} \theta d \varphi^{2}\right)$.
We consider the evolution of massless scalar perturbation. The wave equation for the complex scalar field is , iven by (Hawking \& Ellis (1973)):

$\phi_{; \mu \nu} g^{\mu \nu}-\frac{k^{2}}{\hbar^{2}} \phi=0$,

where $k$ and $\hbar$ are constants. $m=\frac{k}{\hbar}$ represents the mass of the scalar field.

We represent the scalar field into spherical harmonics $\phi=\sum_{l, m} \psi_{m}^{l}(r) e^{-i \omega t} Y_{l}^{m}(\theta, \varphi) / r$ and after some algebra the equation of motion takes the form:

$\psi_{, r_{*} r_{*}}+\left(\omega^{2}-V\right) \psi=0$,

where the black hole potential

$V=f(r)\left[\frac{l(l+1)}{r^{2}}+\frac{2 M}{r^{3}}-\frac{2 Q^{2}}{r^{4}}+\frac{c\left(3 \omega_{q}+1\right)}{r^{3 \omega_{q}+3}}+\frac{k^{2}}{\hbar^{2}}\right]$.

is represented in figure 1. Since we considered a massless scalar field, $k=0$.

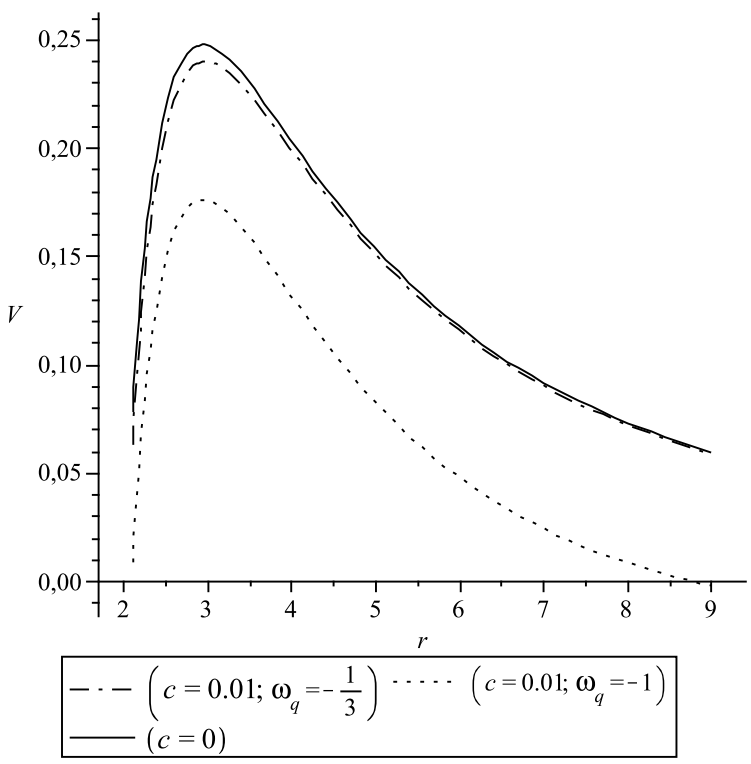

Fig. 1 Black hole's effective potential without quintessence $(c=0)$ and with quintessence $(c=0.01$; $\omega_{q}=-1$ and $\left.\omega_{q}=-1 / 3\right)$

Through this figure, we can see that the nonquintessential potential is higher than the quintessential ones, and the height of the potential decreases with increasing $\omega_{q}$.

\section{Quasinormal modes}

The wave equation ( 5) can be rewritten as:

$\frac{d^{2} \psi}{d r_{*}^{2}}+Q(r) \psi=0$ 
where $Q(r)=\omega^{2}-V$. For a black hole, the QN frequencies correspond to solution of perturbation equation which satisfy the boundary conditions appropriate for purely ingoing waves at the horizon and purely outgoing waves at infinity. Incoming and outgoing waves correspond to the radial solution proportional to $e^{-i \omega r_{*}}$ and $e^{i \omega r_{*}}$, respectively. Only a discrete set of complex frequencies satisfies these conditions.

To evaluate the QN frequencies, we applied the third order WKB approximation method derived by Schutz, Will Schutz \& Will (1985)) and Iyer Iver \& Will (1987) to the above equation and these $\mathrm{QN}$ frequencies are given by (Zhang et al. (2007))

$\omega^{2}=\left[V_{0}+\left(-2 V_{0}^{\prime \prime}\right)^{1 / 2} \tilde{\Lambda}\right]-i\left(n+\frac{1}{2}\right)\left(-2 V_{0}^{\prime \prime}\right)^{1 / 2}[1+\tilde{\Omega}]$,

where

$$
\begin{aligned}
\tilde{\Lambda}= & \frac{1}{\left(-2 V_{0}^{\prime \prime}\right)^{1 / 2}}\left\{\frac{1}{8}\left(\frac{V_{0}^{(4)}}{V_{0}^{\prime \prime}}\right)\left(\frac{1}{4}+\alpha^{2}\right)\right. \\
& \left.-\frac{1}{288}\left(\frac{V_{0}^{\prime \prime \prime}}{V_{0}^{\prime \prime}}\right)^{2}\left(7+60 \alpha^{2}\right)\right\} \\
\tilde{\Omega}= & \frac{1}{-2 V_{0}^{\prime \prime}}\left\{\frac{5}{6912}\left(\frac{V_{0}^{\prime \prime \prime}}{V_{0}^{\prime \prime}}\right)^{4}\left(77+188 \alpha^{2}\right)\right. \\
& -\frac{1}{384}\left(\frac{V_{0}^{\prime \prime \prime 2} V_{0}^{(4)}}{V_{0}^{\prime \prime 3}}\right)\left(51+100 \alpha^{2}\right) \\
& -\frac{1}{288}\left(\frac{V_{0}^{(6)}}{V_{0}^{\prime \prime}}\right)\left(5+4 \alpha^{2}\right)+\frac{1}{288}\left(\frac{V_{0}^{\prime \prime \prime} V_{0}^{(5)}}{V_{0}^{\prime \prime 2}}\right)\left(19+28 \alpha^{2}\right) \\
& \left.+\frac{1}{2304}\left(\frac{V_{0}^{(4)}}{V_{0}^{\prime \prime}}\right)^{2}\left(67+68 \alpha^{2}\right)\right\},
\end{aligned}
$$

$\alpha=n+\frac{1}{2}$, and $V_{0}^{(n)}=\left.\frac{d^{n} V}{d r_{*}^{n}}\right|_{r_{*}=r_{*}\left(r_{p}\right)}$.

Using equation( 8 ), we calculated numerically the QN frequencies of the scalar field perturbation for $M=$ $1, Q=0.1$ without quintessence and with quintessence. The results are shown in the following tables where $l$ is the harmonic angular index, $n$ is the overtone number, $\omega$ is the complex QN frequency and $\omega_{q}$ is the state parameter of the quintessence.

We then plot the behavior of the scalar perturbation for some frequencies. The results are shown in figure 2 .

The QNMs of the Reissner-Nordström black hole surrounded by quintessence were investigated for the Dirac field by Wang et al. (2010) and for the charged massive scalar field by Nijo \& Kuriakose (2009). Comparing these results, we pointed out that the massless scalar field oscillates more rapidly than the Dirac field which oscillates more rapidly than the massive scalar field. But in term of damping, Dirac field damps more rapidly than scalar fields. On the other hand, the massless scalar field damps more rapidly than the massive one. When increasing the state parameter of quintessence $\omega_{q}$, the real part and the absolute value of the imaginary part of $\omega$ are increasing for the scalar

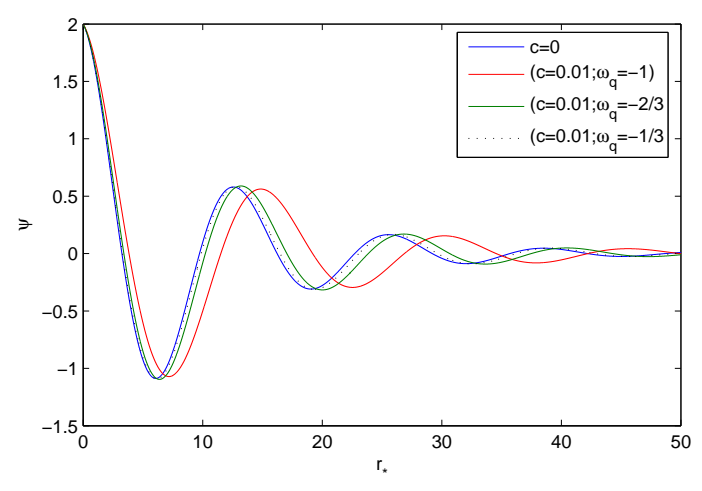

Fig. 2 Behavior of the scalar perturbation for some frequencies $\operatorname{with}(l=2, n=0)$

fields but their variation is negligible for the Dirac field. Moreover, the rate of variation for the massless scalar field is higher than that of the massive one(see Figures (3) and (4).

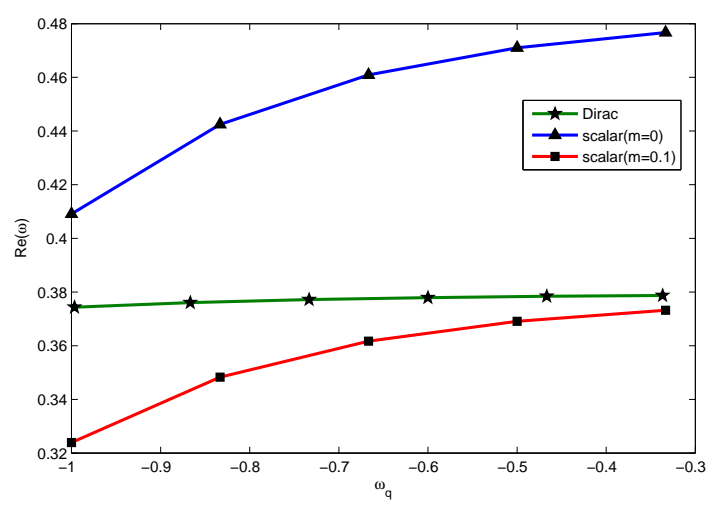

Fig. 3 Comparison of the real parts of $\omega$ when varying $\omega_{q}$ $(l=2, n=0)$

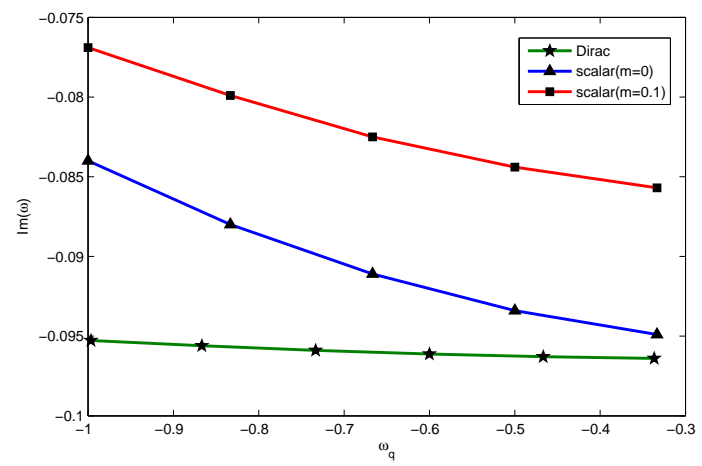

Fig. 4 Comparison of the imaginary parts of $\omega$ when varying $\omega_{q}(l=2, n=0)$ 
Table 1 Quasinormal modes of scalar field perturbation without quintessence

\begin{tabular}{llllcl}
\hline$l$ & $\omega(n=0)$ & $\omega(n=1)$ & $\omega(n=2)$ & $\omega(n=3)$ & $\omega(n=4)$ \\
\hline 2 & $0.4840-0.0969 \mathrm{i}$ & $0.4640-0.2960 \mathrm{i}$ & $0.4326-0.5037 \mathrm{i}$ & $0.3936-0.7162 \mathrm{i}$ & \\
3 & $0.6763-0.0966 \mathrm{i}$ & $0.6616-0.2925 \mathrm{i}$ & $0.6361-0.4944 \mathrm{i}$ & $0.6035-0.7014 \mathrm{i}$ & $0.5651-0.9116 \mathrm{i}$ \\
4 & $0.8688-0.0964 \mathrm{i}$ & $0.8572-0.2911 \mathrm{i}$ & $0.8360-0.4898 \mathrm{i}$ & $0.8080-0.6930 \mathrm{i}$ & $0.7747-0.8998 \mathrm{i}$ \\
5 & $1.0613-0.0964 \mathrm{i}$ & $1.0518-0.2903 \mathrm{i}$ & $1.0338-0.4872 \mathrm{i}$ & $1.0093-0.6878 \mathrm{i}$ & $0.9798-0.8918 \mathrm{i}$ \\
\hline
\end{tabular}

Table 2 Quasinormal modes of scalar field perturbation with quintessence for $c=0.01$ and $k=0$

\begin{tabular}{lllllcl}
\hline $\mathrm{l}$ & $\omega_{q}$ & $\omega(n=0)$ & $\omega(n=1)$ & $\omega(n=2)$ & $\omega(n=3)$ & $\omega(n=4)$ \\
\hline & -1 & $0.4091-0.0840 \mathrm{i}$ & $0.3974-0.2545 \mathrm{i}$ & $0.3779-0.4286 \mathrm{i}$ & $0.3519-0.6049 \mathrm{i}$ & \\
& $-5 / 6$ & $0.4425-0.0880 \mathrm{i}$ & $0.4261-0.2682 \mathrm{i}$ & $0.3998-0.4551 \mathrm{i}$ & $0.3666-0.6459 \mathrm{i}$ & \\
2 & $-2 / 3$ & $0.4609-0.0911 \mathrm{i}$ & $0.4425-0.2783 \mathrm{i}$ & $0.4133-0.4734 \mathrm{i}$ & $0.3772-0.6729 \mathrm{i}$ & \\
& $-1 / 2$ & $0.4710-0.0934 \mathrm{i}$ & $0.4519-0.2854 \mathrm{i}$ & $0.4216-0.4856 \mathrm{i}$ & $0.3842-0.6904 \mathrm{i}$ & \\
& $-1 / 3$ & $0.4767-0.0949 \mathrm{i}$ & $0.4572-0.2900 \mathrm{i}$ & $0.4264-0.4935 \mathrm{i}$ & $0.3883-0.7017 \mathrm{i}$ & \\
\hline & -1 & $0.5747-0.0832 \mathrm{i}$ & $0.5658-0.2508 \mathrm{i}$ & $0.5497-0.4211 \mathrm{i}$ & $0.5281-0.5938 \mathrm{i}$ & $0.5015-0.7680 \mathrm{i}$ \\
& $-5 / 6$ & $0.6199-0.0875 \mathrm{i}$ & $0.6077-0.2648 \mathrm{i}$ & $0.5862-0.4467 \mathrm{i}$ & $0.5585-0.6328 \mathrm{i}$ & $0.5255-0.8215 \mathrm{i}$ \\
3 & $-2 / 3$ & $0.6447-0.0908 \mathrm{i}$ & $0.6311-0.2750 \mathrm{i}$ & $0.6074-0.4646 \mathrm{i}$ & $0.5772-0.6590 \mathrm{i}$ & $0.5415-0.8563 \mathrm{i}$ \\
& $-1 / 2$ & $0.6584-0.0931 \mathrm{i}$ & $0.6443-0.2820 \mathrm{i}$ & $0.6198-0.4766 \mathrm{i}$ & $0.5885-0.6762 \mathrm{i}$ & $0.5516-0.8788 \mathrm{i}$ \\
& $-1 / 3$ & $0.6661-0.0946 \mathrm{i}$ & $0.6517-0.2866 \mathrm{i}$ & $0.6268-0.4844 \mathrm{i}$ & $0.5949-0.6872 \mathrm{i}$ & $0.5574-0.8932 \mathrm{i}$ \\
\hline & -1 & $0.7400-0.0828 \mathrm{i}$ & $0.7329-0.2493 \mathrm{i}$ & $0.7196-0.4176 \mathrm{i}$ & $0.7012-0.5880 \mathrm{i}$ & $0.6786-0.7600 \mathrm{i}$ \\
& $-5 / 6$ & $0.7972-0.0873 \mathrm{i}$ & $0.7875-0.2633 \mathrm{i}$ & $0.7697-0.4426 \mathrm{i}$ & $0.7459-0.6255 \mathrm{i}$ & $0.7174-0.8112 \mathrm{i}$ \\
4 & $-2 / 3$ & $0.8285-0.0907 \mathrm{i}$ & $0.8178-0.2736 \mathrm{i}$ & $0.7982-0.4602 \mathrm{i}$ & $0.7722-0.6511 \mathrm{i}$ & $0.7413-0.8452 \mathrm{i}$ \\
& $-1 / 2$ & $0.8459-0.0930 \mathrm{i}$ & $0.8348-0.2807 \mathrm{i}$ & $0.8145-0.4722 \mathrm{i}$ & $0.7876-0.6681 \mathrm{i}$ & $0.7556-0.8674 \mathrm{i}$ \\
& $-1 / 3$ & $0.8557-0.0945 \mathrm{i}$ & $0.8444-0.2852 \mathrm{i}$ & $0.8237-0.4800 \mathrm{i}$ & $0.7963-0.6791 \mathrm{i}$ & $0.7638-0.8816 \mathrm{i}$ \\
\hline & -1 & $0.9051-0.0827 \mathrm{i}$ & $0.8992-0.2485 \mathrm{i}$ & $0.8879-0.4157 \mathrm{i}$ & $0.8721-0.5846 \mathrm{i}$ & $0.8523-0.7550 \mathrm{i}$ \\
& $-5 / 6$ & $0.9745-0.0872 \mathrm{i}$ & $0.9664-0.2626 \mathrm{i}$ & $0.9514-0.4403 \mathrm{i}$ & $0.9306-0.6210 \mathrm{i}$ & $0.9054-0.8044 \mathrm{i}$ \\
5 & $-2 / 3$ & $1.0124-0.0906 \mathrm{i}$ & $1.0035-0.2729 \mathrm{i}$ & $0.9870-0.4578 \mathrm{i}$ & $0.9642-0.6462 \mathrm{i}$ & $0.9368-0.8377 \mathrm{i}$ \\
& $-1 / 2$ & $1.0335-0.0930 \mathrm{i}$ & $1.0243-0.2800 \mathrm{i}$ & $1.0071-0.4698 \mathrm{i}$ & $0.9836-0.6631 \mathrm{i}$ & $0.9553-0.8598 \mathrm{i}$ \\
& $-1 / 3$ & $1.0454-0.0945 \mathrm{i}$ & $1.0360-0.2845 \mathrm{i}$ & $1.0186-0.4775 \mathrm{i}$ & $0.9946-0.6740 \mathrm{i}$ & $0.9658-0.8739 \mathrm{i}$ \\
\hline
\end{tabular}

Let's rescale the time coordinate into EddingtonFinkelstein coordinate(Zhai \& Liu (2010))

$t=T \pm r_{*}$,

where the signs + and - represent ingoing and outgoing particles, respectively. The tortoise coordinate $r_{*}$ is defined as

$\frac{d r_{*}}{d r}=f(r)^{-1}$.

In the following, the study is restricted to the outgoing particle radiated from the black hole horizon.

The background metric can then transform to

$d s^{2}=-f(r) d T^{2}+2 d T d r+r^{2}\left(d \theta^{2}+\sin ^{2} \theta d \varphi^{2}\right)$.

\section{Hawking radiation}

Actually, the normalization factor related to the density of quintessence, $c$, is smaller than 0.001 (Zhang \& Gui (2006)). Therefore, the contribution to the metric background due to the presence of quintessence can be treated as a perturbation.

We regard the quintessence as perturbation leading to a small modification of the horizon radii and we put then the quintessential horizon radii $R_{ \pm}$in the form

$R_{ \pm}=r_{ \pm}+\epsilon_{ \pm}$

Substituting this expression into Eq.(11), we obtain

$$
\begin{aligned}
& 1-\frac{2 M}{r_{ \pm}}\left(1-\frac{\epsilon_{ \pm}}{r_{ \pm}}\right)+\frac{Q^{2}}{r_{ \pm}^{2}}\left(1-\frac{2 \epsilon_{ \pm}}{r_{ \pm}}\right) \\
& -c r_{ \pm}^{-3 \omega_{q}-1}\left(1-\left(1+3 \omega_{q}\right) \frac{\epsilon_{ \pm}}{r_{ \pm}}\right)=0
\end{aligned}
$$

The metric obtained is a Vaidya-Bonner like metric(Niu \& Liu (2010) ) and can represent a Vaidya-Bonner black hole surrounded by quintessence.

The apparent horizon of this metric is given by the following equation

$1-\frac{2 M}{r}+\frac{Q^{2}}{r^{2}}-\frac{c}{r^{3 \omega_{q}+1}}=0$.

In the absence of quintessence $(c=0)$, this equation gives two solutions $r_{ \pm}=M \pm \sqrt{M^{2}-Q^{2}}$. whinch gives us

$\epsilon_{ \pm} \simeq \frac{c r_{ \pm}^{1-3 \omega_{q}}}{r_{ \pm}-r_{\mp}}$

in first approximation.

The radial null geodesic is given by

$\dot{r}=\frac{d r}{d T}=\frac{1}{2}\left(1-\frac{2 M}{r}+\frac{Q^{2}}{r^{2}}-\frac{c}{r^{3 \omega_{q}+1}}\right)$. 
When a particle of energy $\omega$ is radiated from the black hole, it transforms to

$\dot{r}=\frac{1}{2}\left(1-\frac{2(M-\omega)}{r}+\frac{Q^{2}}{r^{2}}-\frac{c}{r^{3 \omega_{q}+1}}\right)$.

The imaginary part of the action is

$\operatorname{Im} S=\operatorname{Im} \int P_{r} d r=\operatorname{Im} \iint d P_{r} d r=\operatorname{Im} \iint \frac{d H}{\dot{r}} d r$

where we have used the Hamilton equation $\frac{d H}{d P_{r}}=\dot{r}$, $H=M-\omega^{\prime} \Rightarrow d H=-d \omega^{\prime}$. The imaginary part of the action takes then the form

$$
\begin{aligned}
\operatorname{Im} S & =\operatorname{Im} \int_{M}^{M-\omega} \int \frac{2 d r}{1-\frac{2 M}{r}+\frac{Q^{2}}{r^{2}}-\frac{c}{r^{3 \omega_{q}+1}}}\left(-d \omega^{\prime}\right) \\
& =\operatorname{Im} \int \frac{2 \omega r^{2} d r}{\left(r-R_{+}\right)\left(r-R_{-}\right)} .
\end{aligned}
$$

We used the tunnelling method of Parikh \& Wilczek (2000) to evaluate the integral over $r$ and obtain

$\operatorname{Im} S=\frac{2 \pi R_{+}^{2}}{R_{+}-R_{-}} \omega$.

Using the WKB approximation, the rate of radiation is expressed as

$\Gamma \propto e^{-2 \operatorname{Im} S}=e^{-\beta \omega}$

where $\beta$ is the Boltzmann factor with inverse temperature expressed as

$\beta=\frac{1}{T}=\frac{4 \pi r_{+}^{2}}{r_{+}-r_{-}}\left(1+\frac{c\left(r_{+}^{-3 \omega_{q}}\left(r_{+}-2 r_{-}\right)-r_{-}^{1-3 \omega_{q}}\right)}{\left(r_{+}-r_{-}\right)^{2}}\right)$.

Explicitly, we plot the variation of the Boltzmann factor with respect to the state parameter of quintessence. Its behavior is represented in Figure 5.

For a black hole with a charge such as ReissnerNordström black hole, the emitted particles can be charged. Thus, not only energy conservation but also electric charge conservation should be considered. Then, the radial null geodesics transforms to

$\dot{r}=\frac{1}{2}\left(1-\frac{2(M-\omega)}{r}+\frac{(Q-q)^{2}}{r^{2}}-\frac{c}{r^{3 \omega_{q}+1}}\right)$,

where $q$ is the charge of the emitted particle.

The electromagnetic potential becomes

$A_{t}=\frac{Q-q}{r}$

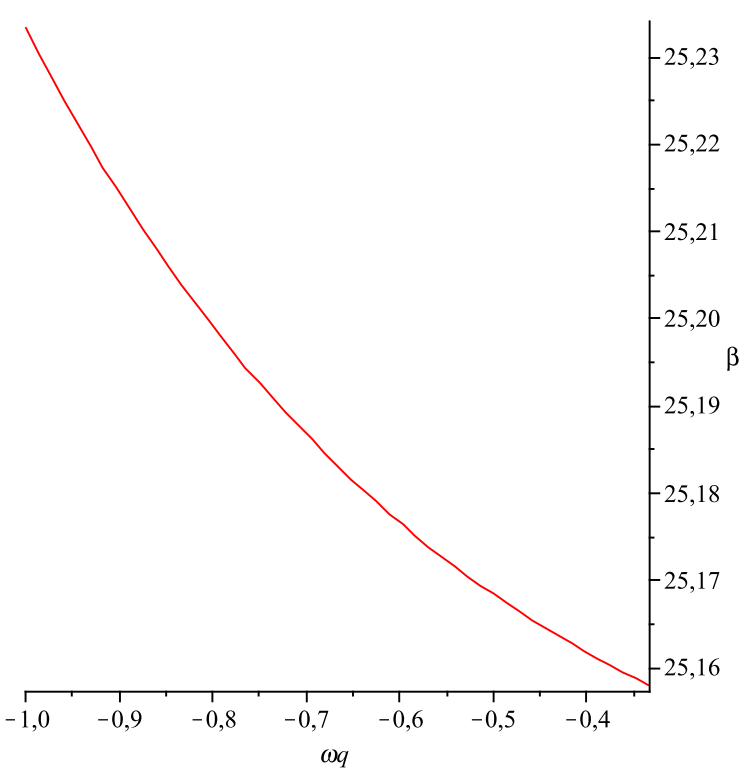

Fig. 5 Variation of the Boltzmann factor with the state parameter of quintessence.

The imaginary part of the action for the massive charged particle is (Jiang \& Wu (2006)):

$$
\begin{aligned}
\operatorname{ImS} & =\operatorname{Im} \int_{t_{i}}^{t_{f}}\left(L-P_{A_{t}} \dot{A}_{t}\right) d t \\
& =\operatorname{Im} \int_{r_{i e}}^{r_{f e}}\left(P_{r} \dot{r}-P_{A_{t}} \dot{A}_{t}\right) \frac{d r}{\dot{r}} \\
& =\operatorname{Im} \int_{r_{i e}}^{r_{f e}}\left[\int_{(0,0)}^{\left(P_{r}, P_{A_{t}}\right)}\left(\dot{r} d P_{r}^{\prime}-\dot{A}_{t} d P_{A_{t}}^{\prime}\right)\right] \frac{d r}{\dot{r}},
\end{aligned}
$$

where $r_{i e}$ and $r_{f e}$ represent the localization of the event horizon before and after the particle with energy $\omega$ and charge $q$ tunnels out. $\dot{r}$ and $\dot{A}_{t}$ are given by the Hamilton's canonical equation of motion

$$
\begin{cases}\dot{r}=\left.\frac{d H}{d P_{r}}\right|_{\left(r ; A_{t}, P_{A_{t}}\right)}, & \left.d H\right|_{\left(r ; A_{t}, P_{A_{t}}\right)}=d(M-\omega) \\ \dot{A}_{t}=\left.\frac{d H}{d P_{A_{t}}}\right|_{\left(A_{t} ; r, P_{r}\right)}, & \left.d H\right|_{\left(A_{t} ; r, P_{r}\right)}=\frac{Q-q}{r} d(Q-q) .\end{cases}
$$

Substituting equations ( 22) and (25) into (24), we obtain

$$
\begin{aligned}
& \operatorname{Im} S=\operatorname{Im} \int_{(M, Q)}^{(M-\omega, Q-q)}\left[\left.d H\right|_{\left(r ; A_{t}, P_{A_{t}}\right)}-\left.d H\right|_{\left(A_{t} ; r, P_{r}\right)}\right] \frac{d r}{\dot{r}} \\
& =\operatorname{Im} \int_{r_{i e}}^{r_{f e}} \int_{(M, Q)}^{(M-\omega, Q-q)} \frac{2\left[d\left(M-\omega^{\prime}\right)-\frac{Q-q^{\prime}}{r} d\left(Q-q^{\prime}\right)\right] d r}{\left(1-\frac{2\left(M-\omega^{\prime}\right)}{r}+\frac{\left(Q-q^{\prime}\right)^{2}}{r^{2}}-\frac{c}{r^{3 \omega_{q}+1}}\right)} .
\end{aligned}
$$

Using the method of Parikh \& Wilczek (2000), we can get

$\operatorname{Im} S=-\operatorname{Im} \int_{r_{i e}}^{r_{f e}}(i \pi r) d r=\frac{\pi}{2}\left(r_{i e}^{2}-r_{f e}^{2}\right)$. 
Using the WKB approximation, we can get the tunnelling rate of radiation

$\Gamma \propto e^{-2 \operatorname{Im} S}=e^{\pi\left(r_{f e}^{2}-r_{i e}^{2}\right)}=e^{\Delta S_{E H}}$,

where $\Delta S_{E H}$ denotes the change of Bekenstein-Hawking entropy at the even horizon before and after the particle tunnelled out, expressed as

$\Delta S_{E H}=\pi\left(R_{+}^{2}(M-\omega, Q-q)-R_{+}^{2}(M, Q)\right)$,

where

$R_{+}^{2}(x, y)=\left(x+\sqrt{x^{2}-y^{2}}\right)^{2}+\frac{c\left(x+\sqrt{x^{2}-y^{2}}\right)^{2-3 \omega_{q}}}{\sqrt{x^{2}-y^{2}}}$.

The change of Bekenstein-Hawking entropy at the even horizon can then be written as

$\Delta S_{E H}=\Delta S_{0}+\Delta S_{q}$,

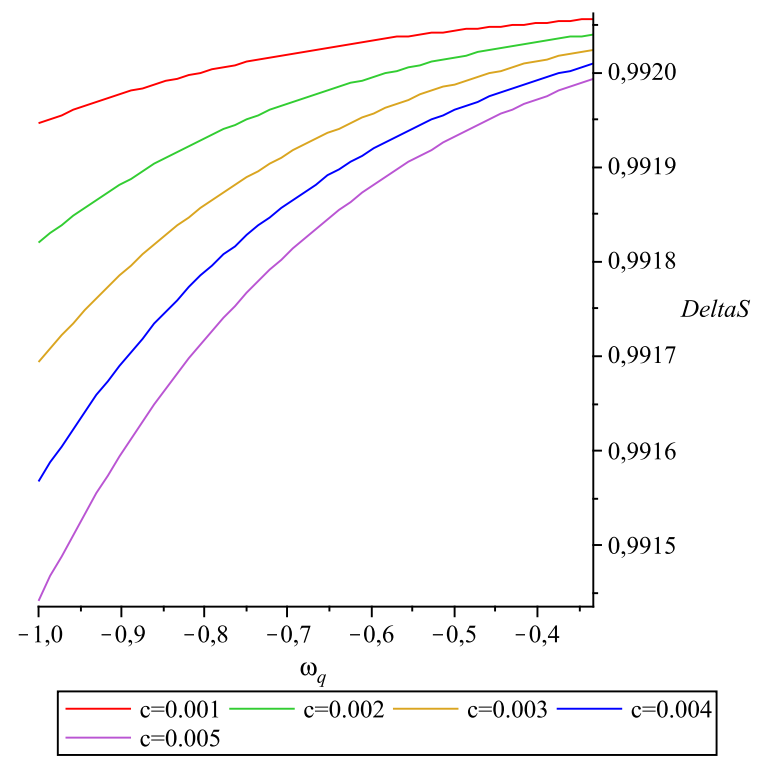

where

$$
\begin{gathered}
\Delta S_{0}=\pi\left[\left(M-\omega+\sqrt{(M-\omega)^{2}-(Q-q)^{2}}\right)^{2}\right. \\
\left.-\left(M+\sqrt{(M)^{2}-(Q)^{2}}\right)^{2}\right], \\
\Delta S_{q}=\pi c\left[\frac{\left(M-\omega+\sqrt{(M-\omega)^{2}-(Q-q)^{2}}\right)^{2-3 \omega_{q}}}{\sqrt{(M-\omega)^{2}-(Q-q)^{2}}}\right. \\
\left.-\frac{\left(M+\sqrt{(M)^{2}-(Q)^{2}}\right)^{2-3 \omega_{q}}}{\sqrt{(M)^{2}-(Q)^{2}}}\right],
\end{gathered}
$$

$\Delta S_{0}$ is the free variation of the entropy and $\Delta S_{q}$ is the contribution of the entropy variation due to the quintessence.

Supposing that the mass and charge of the black hole are uniformly distributed and considering that the black hole radiates particles with energy and charge proportional to the total mass and charge, respectively, with the same coefficient of proportionality $a$,

$\omega=a M, \quad q=a Q, \quad a<<1$,

the variation of entropy can be written as

$$
\begin{aligned}
\Delta S=\Delta S_{0}+\Delta S_{q} \\
\simeq \quad-a \pi\left[2\left(M+\sqrt{M^{2}-Q^{2}}\right)^{2}\right. \\
\left.\quad+\left(1-\omega_{q}\right) c \frac{\left(M+\sqrt{M^{2}-Q^{2}}\right)^{2-3 \omega_{q}}}{\sqrt{M^{2}-Q^{2}}}\right] .
\end{aligned}
$$

Its behavior is plotted in Figure 6.

Through this figure, we can see that the variation of entropy is decreasing when decreasing $\omega_{q}$. We can also see that it is decreasing when increasing $c$.

Fig. 6 Variation of the entropy versus the state parameter of quintessence.

\section{Summary and Conclusion}

In summary, QNMs of a scalar field perturbation around a Reissner-Nordström black hole were evaluated using the third order WKB approximation. The results of table 1 are obtained without the presence of quintessence while those of table 2 are obtained under the presence of quintessence for some values of the state parameter of the quintessence. The Boltzmann factor with inverse temperature was also derived and its behavior is plotted when varying the state parameter of quintessence. The behavior of the variation of entropy is also plotted when varying $c$ and $\omega_{q}$, respectively.

Through the above tables, we can remark that the absolute values of the imaginary parts of the quasinormal frequencies under quintessence are smaller compared to those without quintessence, for fixed set of $l$ and $n$. Moreover, we can remark through table 2 that these values decrease when decreasing $\omega_{q}$. From the variation of the Boltzmann factor plotted bellow, we can see that it is increasing when decreasing $\omega_{q}$. From the behavior of the variation of entropy with respect to $c$ and $\omega_{q}$, respectively, we can remark that this variation of entropy is decreasing when increasing $c$ or when decreasing $\omega_{q}$, denoting that the rate of radiation is decreasing. Decreasing $\omega_{q}$ for fixed $c$, or increasing $c$ for fixed $\omega_{q}$ means increasing the density of quintessence. Thus, we can conclude that when increasing the density of quintessence surrounding the Reissner-Nordström black hole, the QNMs damp more slowly and the black hole radiates at slower rate. 


\section{References}

Cardenas, R., Tame, G., Yoelsy, L., Osmel, M., \& Quiros, I. 2003, Phys. Rev. D, 67, 083501

Cardoso, V., Konoplya, R., \& Lemos, J. P. S. 2003, Phys. Rev. D, 68, 044024

Goncharov, Yu. P. \& Firsova, N. E. 2010, Nucl. Phys. B, 486, 371

Guo, Z., Piao, Y., Zhang, X., \& Zhang, Y. 2005, Phys. Lett. B, 608, 177

Hawking, S. W., \& Ellis, G. F. R. 1973, The large scale structure of space-time, Cambridge University Press, Cambridge

Hod, S., \& Piran, T. 1998, Phys. Rev. D, 58, 024017

Iyer, S., \& Will, C. M. 1987 Phys. Rev. D, 35, 3621

Iyer, S., \& Will, C. M. 1987, Phys. Rev. D, 35, 3632

Jiang, Q.-Q. \& Wu, S.-Q. 2006, Phys. Lett. B, 635, 151

Kiselev, V. V. 2003, Class. Quant. Grav., 20, 1187

Kokkotas, K. D. 1993, Nuovo Cimento B, 108, 991

Kokkotas, K. D., \& Schutz, B. F. 1988, Phys. Rev. D, 37, 3378

Konoplya, R. A. 2002, Phys. Rev. D, 66, 084007

Kunz M., \& Domenico S. 2006, Phys. Rev. D, 74, 123503

Mahamat, S., Bouetou, B. T., \& Kofane, T. C. 2009, Chin. Phys. Lett., 26, 109802

Mukhanov, V. 2005, Physical Foundations of Cosmology, Cambridge University Press, New York

Nijo, V. \& Kuriakose, V. C. 2009, Gen. Rel. Grav., 41, 1249

Niu Z.-F., \& Liu W. 2010, Research Astron. Astrophys., 10, 33

Parikh, M. K. \& Wilczek, F. 2000, Phys. Rev. Lett., 85, 5042

Schutz, B. F., \& Will, C. M. 1985, Astrophys. J., 291, L33

Seidel, E., \& Iyer S. 1990, Phys. Rev. D, 41, 374

Shuang-Yang, Z. 2008, Phys. Lett. B, 660, 7

Varun, S., \& Wang, L., 2000, Phys. Rev. D, 62, 103517

WANG, C.-Y., ZHANG Yu, GUI, Y.-X., \& LÜ, J.-B. 2010, Commun. Theor. Phys., 53, 882

Xia, J., Feng, B., \& Zhang, X. 2006, Phys. Rev. D, 74, 123503

Yang, R., \& Gao, X. 2009, Chin. Phys. Lett., 26, 089501

Yu Zhang, \& Gui, Y. X. 2006, Class. Quant. Grav., 23, 6141

Yu Zhang, Gui, Y. X., Fei Yu, \& Feng Lin Li 2007, Gen. Rel. Grav., 39, 1003

Zhai Z., \& Liu W. 2010, Astrophys. Space Sci., 325, 63

Zhao, R., Zhang, L.-C. \& Li, H.-F. 2010, Commun. Theor. Phys., 53, 499 